\title{
Evaluation of the CellaVision Advanced RBC Application for Detecting Red Blood Cell Morphological Abnormalities
}

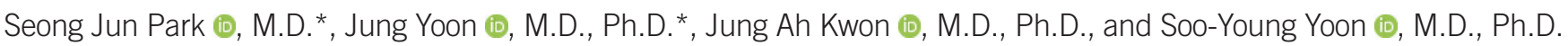 \\ Department of Laboratory Medicine, Korea University Guro Hospital, Seoul, Korea
}

Background: The Advanced RBC Application of the CellaVision DM9600 system (CellaVision $A B$, Lund, Sweden) automatically characterizes and classifies red blood cells (RBCs) into 21 morphological categories based on their size, color, shape, and inclusions. We evaluated the diagnostic performance of the CellaVision Advanced RBC Application with respect to the classification and grading of RBC morphological abnormalities in accordance with the 2015 International Council for Standardization in Haematology (ICSH) guidelines.

Methods: A total of 223 samples, including 123 with RBC morphological abnormalities and 100 from healthy controls, were included. Seven RBC morphological abnormalities and their grading obtained with CellaVision DM9600 pre- and post-classification were compared with the results obtained using manual microscopic examination. The grading cut-off percentages were determined in accordance with the 2015 ICSH guidelines. The sensitivity and specificity of the CellaVision DM9600 system were evaluated using the manual microscopic examination results as a true positive.

Results: In pre-classification, $>90 \%$ sensitivity was observed for target cells, tear drop cells, and schistocytes, while $>90 \%$ specificity was observed for acanthocytes, spherocytes, target cells, and tear drop cells. In post-classification, the detection sensitivity and specificity of most RBC morphological abnormalities increased, except for schistocytes (sensitivity) and acanthocytes (specificity). The grade agreement rates ranged from 35.9\% (echinocytes) to $89.7 \%$ (spherocytes) in pre-classification and from $46.2 \%$ (echinocytes) to $90.1 \%$ (spherocytes) in post-classification. The agreement rate of samples with withinone grade difference exceeded $90 \%$ in most categories, except for schistocytes and echinocytes.

Conclusions: The Advanced RBC Application of CellaVision DM9600 is a valuable screening tool for detecting RBC morphological abnormalities.

Key Words: Red blood cell, Morphology, Advanced RBC Application, CellaVision DM9600, International Council for Standardization in Haematology guidelines
Received: February 10, 2020

Revision received: March 24, 2020

Accepted: August 6, 2020

\section{Corresponding author:}

Soo-Young Yoon, M.D., Ph.D.

Department of Laboratory Medicine,

Korea University College of Medicine, 148

Gurodong-ro, Guro-gu, Seoul 08308, Korea

Tel: +82-2-2626-3246

Fax: +82-2-2626-1465

E-mail: labmd@korea.ac.kr

\section{Co-corresponding author:}

Jung Ah Kwon, M.D., Ph.D.

Department of Laboratory Medicine,

Korea University College of Medicine, 148

Gurodong-ro, Guro-gu, Seoul 08308, Korea

Tel: +82-2-2626-3246

Fax: +82-2-2626-1465

E-mail: jakwon83@hanmail.net

*These authors contributed equally to this work.

\section{(c) (i) (8)}

(c) Korean Society for Laboratory Medicine

This is an Open Access article distributed under the terms of the Creative Commons Attribution Non-Commercial License (https://creativecommons.org/licenses/by-nc/4.0) which permits unrestricted non-commercial use, distribution, and reproduction in any medium, provided the original work is properly cited.

\section{INTRODUCTION}

Microscopic examination of peripheral blood (PB) smear provides important information to support the diagnosis of hematological diseases [1, 2]. However, manual microscopic examina- tion of PB smears is labor-intensive, requires highly trained personnel, and may result in observer-specific bias. CellaVision DM9600 (CellaVision AB, Lund, Sweden) is an automated image analysis system that classifies white blood cells (WBCs) and red blood cells (RBCs) based on their morphology. This system 
detects WBC morphology with greater accuracy and precision than that achieved by manual microscopic examination [2-4]. Several studies evaluated the performance of this system ; however, these have mainly focused on WBCs and blast differentiation [2-4].

The Advanced RBC Application is a new software in the CellaVision DM9600 system that can be used to perform advanced morphological analyses of RBCs. The software is programed to automatically pre-classify the RBCs into 21 morphological categories based on parameters including size, color, shape, and inclusions. Based on these morphological categories, the RBCs can be graded semi-quantitatively (using four grades) or quantitatively as percentage of RBCs. Although a few studies have evaluated the performance of this software for detecting and grading RBCs $[5,6]$, to our knowledge, only one study have evaluated the grading of RBC morphological features according to the 2015 International Council for Standardization in Haematology (ICSH) guidelines [7, 8].

We evaluated the performance of the CellaVision Advanced RBC Application for the characterization of seven RBC morphological abnormalities, namely acanthocytes, echinocytes, elliptocytes, schistocytes, spherocytes, target cells, and tear drop cells. Ovalocytes and helmet cells were incorporated into elliptocytes and schistocytes, respectively. The cut-off percentages for the detection and grading of RBC morphological abnormalities were applied in accordance with the 2015 ICSH guidelines [7].

\section{MATERIALS AND METHODS}

\section{Samples}

This retrospective study was conducted in Korea University Guro Hospital, Seoul, Korea, from October 2018 to September 2019. A total of 223 PB samples, including 100 samples from healthy controls with normal complete blood count results and 123 samples from patients with RBC morphological abnormalities, were included. Some patients with RBC morphological abnormalities had various underlying diseases, including iron deficiency anemia ( $\mathrm{N}=14)$, microangiopathic hemolytic anemia (MAHA; $N=21$ ), hereditary spherocytosis ( $\mathrm{N}=2)$, autoimmune hemolytic anemia $(\mathrm{N}=2)$, and liver disease $(\mathrm{N}=15)$. Thin PB smear slides were prepared using ethylenediaminetetraacetic acid (EDTA)-anticoagulated venous blood samples. The slides were immediately stained with Wright-Giemsa stain using an automated DxH Slidemaker Stainer (DxH SMS; Beckman Coulter Inc., Miami, FL, USA). This study was approved by the Institutional Review Board of Korea University Guro Hospital (2018GR0338), which waived the need for informed consent.

\section{Manual microscopic examination}

Manual microscopic examination of RBC morphology was performed using a model BX53 microscope (Olympus Corporation, Tokyo, Japan). A minimum of 1,000 RBCs was examined in accordance with the 2015 ICSH guidelines [7, 9]. Two experienced hematopathologists independently scanned 1,000 RBCs (4-5 high-power fields using a $100 \times$ objective lens). The percentage of RBCs with morphological abnormalities was defined as the mean percentage of RBCs measured by each hematopathologist.

\section{Automated analysis using CellaVision DM9600}

CellaVision DM9600 uses artificial neural networks to locate, identify, and categorize RBCs. This system automatically categorizes RBCs (pre-classification), and the user subsequently manually verifies or re-classifies the RBCs before signing off the sample (post-classification). The CellaVision Advanced RBC Application uses a $50 \times$ objective lens to select and scan one zone of the slide for the analysis of approximately 2,000 to 4,000 RBCs per blood smear $[5,6]$. The RBC category results are reported semiquantitatively in four flag levels (0, 1, 2, and 3 ) based on cut-off percentages established by each laboratory. The results may also be represented quantitatively as a percentage count of all analyzed RBCs (Fig. 1). We used software version 7.0.1 for the evaluation.

\section{RBC morphological category cut-offs}

The 2015 ICSH guidelines recommend two-tiered grading of RBC morphologies (2+, moderate and 3+, many; except for schistocytes), so that only clinically important results are reported [7]. We defined the cut-off percentages for these morphological categories as $1-5 \%$ of RBCs for grade 1+ (few/rare), 5-20\% for grade $2+$, and $>20 \%$ for grade $3+$, in accordance with the 2015 ICSH guidelines [7].

The 2015 ICSH guidelines recommend a different grading system and cut-off percentages for schistocytes: $<1 \%$ for grade $1+, 1-2 \%$ for grade $2+$ and $>2 \%$ for grade $3+$. Schistocytes should be reported even when graded $1+$, which corresponds to $<1 \%$ of RBCs, as this observation is clinically significant, even for low numbers of cells $[7,10,11]$. To establish a lower cut-off percentage of $1+$, we used the distribution statistics of the control population [5]. The cut-off value for schistocytes was determined using a nonparametric method of percentile calculation; the 97.5 percentile of the normal healthy population was $0.2 \%$ 


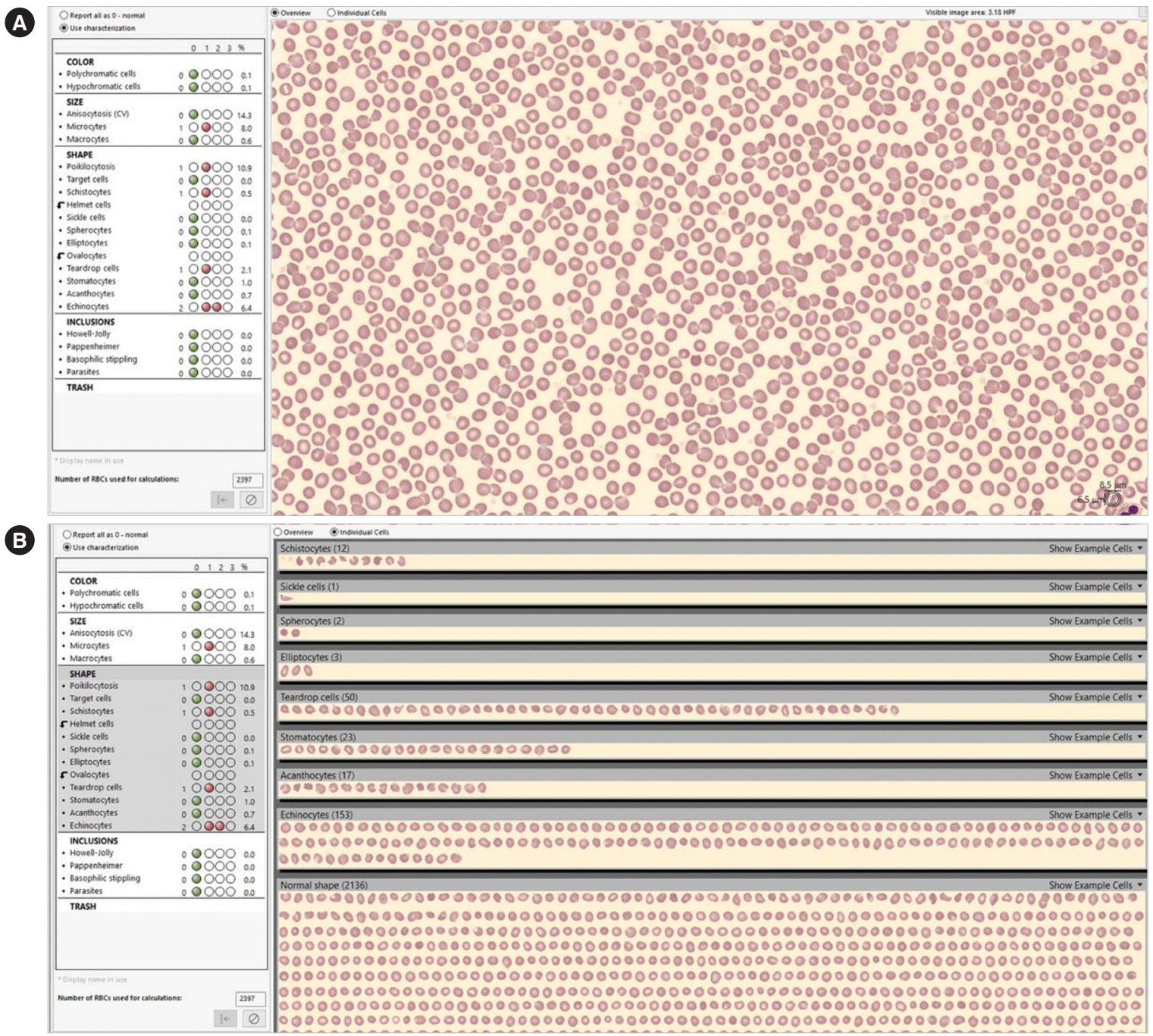

Fig. 1. Overview of the CellaVision DM9600 Advanced RBC Application. (A) The software provides an overview image using the RBC morphological category results, which are graded semi-quantitatively in four flag levels. (B) Image depicting individual cells categorized into each RBC morphological abnormality.

Abbreviation: RBC, red blood cell.

of RBCs for manual microscopic examination and $0.5 \%$ of RBCs for the DM9600. Therefore, the schistocyte grade cut-off percentages were grade $1+:<0.2-1 \%$, grade $2+:<1-2 \%$, and grade $3+$ : $>2 \%$ for microscopic examination and grade $1+:<0.5-$ $1 \%$, grade $2+:<1-2 \%$, and grade $3+:>2 \%$ for DM9600.

We defined 'positive' RBC morphological abnormalities as reportable, clinically important RBC morphological abnormalities, represented by grade 1-3 for schistocytes and grade 2-3 for the other categories. 'Negative' RBC morphological abnormalities were considered for detected values below the cut-off percentage for grades 1 and 2 for schistocytes and other categories, respectively.

\section{Statistical analyses}

The sensitivity and specificity of the CellaVision DM9600 system with respect to RBC morphological abnormalities were evaluated using the manual microscopic examination results as a true positive. The following represented the true positive for each category: RBC morphological abnormalities with manual microscopic examination grade 1-3 for schistocytes and grade 2-3 for the other categories. Moreover, area under the receiver operating characteristic curve (AUC) was assessed to measure the overall diagnostic ability of the CellaVision DM9600 with respect to the detection of reportable RBC morphological abnormalities. The agreement between the grades determined by CellaVision DM9600 analysis and manual microscopic examination was com- 
pared by measuring grade agreement rate and one grade difference agreement rate (the agreement rate of samples with a withinone grade difference) with 95\% confidence intervals (Cls). Statistical analyses were conducted using Microsoft Excel 2010 (Microsoft Corp., Redmond, WA, USA).

\section{RESULTS}

The numbers of samples detected for each RBC morphological abnormality category using manual microscopic examination are shown in Table 1.

\section{Reportable RBC abnormality detection sensitivity and specificity}

Pre-classification detection-positive (reportable) RBC morphological abnormality sensitivity varied across the categories compared with manual microscopic examination. The pre-classification sensitivity ranged from 10.0\% (acanthocytes) to 100\% (tar-

Table 1. Number of samples per RBC morphological abnormality detected by manual microscopic examination of 223 samples

\begin{tabular}{lccc}
\hline $\begin{array}{l}\text { RBC morphological } \\
\text { abnormality }\end{array}$ & \multicolumn{3}{c}{$\begin{array}{c}\text { Nof samples (multiple categories } \\
\text { per sample are possible) }\end{array}$} \\
\cline { 2 - 4 } & Total & Grade 1 & Grade 2 or 3 \\
\hline Acanthocytes & 43 & 33 & 10 \\
Echinocytes & 62 & 53 & 9 \\
Elliptocytes & 87 & 63 & 24 \\
Schistocytes & 67 & 58 & 9 \\
Spherocytes & 21 & 12 & 9 \\
Target cells & 12 & 7 & 5 \\
Tear drop cells & 29 & 17 & 12 \\
\hline
\end{tabular}

Abbreviation: RBC, red blood cell. get cells and tear drop cells). In some categories, including schistocytes, target cells, and tear drop cells, the sensitivity exceeded $90 \%$, while the sensitivity for spherocytes and acanthocytes was $<20 \%$. Specificity ranged from $58.3 \%$ (schistocytes) to $99.5 \%$ (spherocytes) (Table 2).

When the CellaVision pre-classification results were reviewed by the user (post-classification), the sensitivity for most RBC morphological abnormalities increased, except for schistocytes. Increased post-classification specificity was observed for echinocytes, schistocytes, and target cells, whereas decreased postclassification specificity was observed for acanthocytes and elliptocytes (Table 2). Consistent with the sensitivity and specificity results, the pre- and post-classification AUC values were both highest for tear drop cells and lowest for acanthocytes.

\section{Agreement analysis of RBC morphological abnormality grades}

The grade agreement rates ranged from 35.9\% (echinocytes) to $89.7 \%$ (spherocytes) in pre-classification and from $46.2 \%$ (echinocytes) to $90.1 \%$ (spherocytes) in post-classification (Table 3). In addition, we analyzed the agreement rate of samples with a within-one grade difference between manual microscopy and CellaVision DM9600. The pre- and post-classification one grade difference agreement rate was $>90 \%$ in most categories, except for schistocytes and echinocytes.

\section{DISCUSSION}

Recently, the rapid development of automated image analysis system for morphological assessment of blood smear has been noted [12]. Various image analysis system from several manufacturers are available and the need for standardization has been

Table 2. Sensitivity, specificity, and AUC of all RBC morphological categories

\begin{tabular}{|c|c|c|c|c|c|c|c|c|}
\hline \multirow[b]{2}{*}{ Poikilocytes } & \multicolumn{2}{|c|}{ Cut-off (\%) } & \multicolumn{2}{|c|}{ Sensitivity (\%) (95\% Cl) } & \multicolumn{2}{|c|}{ Specificity (\%) (95\% Cl) } & \multicolumn{2}{|c|}{ AUC (95\% Cl) } \\
\hline & $\begin{array}{c}\text { Manual } \\
\text { microscopy }\end{array}$ & DM 9600 & $\begin{array}{c}\text { Pre- } \\
\text { classification }\end{array}$ & $\begin{array}{c}\text { Post- } \\
\text { classification }\end{array}$ & $\begin{array}{c}\text { Pre- } \\
\text { classification }\end{array}$ & $\begin{array}{c}\text { Post- } \\
\text { classification }\end{array}$ & $\begin{array}{c}\text { Pre- } \\
\text { classification }\end{array}$ & $\begin{array}{c}\text { Post- } \\
\text { classification }\end{array}$ \\
\hline Acanthocytes & $>5$ & $>5$ & $10.0(0.3-44.5)$ & $30.0(6.7-65.3)$ & $98.1(95.3-99.5)$ & $96.2(92.7-98.4)$ & $0.54(0.47-0.61)$ & $0.63(0.56-0.70)$ \\
\hline Echinocytes & $>5$ & $>5$ & $88.9(51.8-99.7)$ & $100(66.4-100)$ & $74.3(67.9-80.0)$ & $79.4(73.4-84.7)$ & $0.82(0.76-0.87)$ & $0.90(0.85-0.93)$ \\
\hline Elliptocytes & $>5$ & $>5$ & $83.3(62.6-95.3)$ & $91.7(73.0-99.0)$ & $86.4(80.9-90.9)$ & $83.9(78.1-88.7)$ & $0.85(0.80-0.89)$ & $0.88(0.83-0.92)$ \\
\hline Schistocytes & $>0.2$ & $>0.5$ & $98.5(92.0-100)$ & $92.5(83.4-97.5)$ & $58.3(50.2-66.2)$ & $71.8(64.0-78.7)$ & $0.78(0.72-0.84)$ & $0.82(0.77-0.87)$ \\
\hline Spherocytes & $>5$ & $>5$ & $11.1(0.3-48.3)$ & $33.3(7.5-70.1)$ & $99.5(97.4-100)$ & $99.5(97.4-100)$ & $0.55(0.49-0.62)$ & $0.66(0.60-0.73)$ \\
\hline Target cells & $>5$ & $>5$ & $100(47.8-100)$ & $100(47.8-100)$ & 96.3 (92.9-98.4) & $97.7(94.7-99.3)$ & $0.98(0.95-0.99)$ & $0.99(0.96-1.00)$ \\
\hline Tear drop cells & $>5$ & $>5$ & $100(73.5-100)$ & $100(73.5-100)$ & $99.1(96.6-99.9)$ & $99.1(96.6-99.9)$ & $1.00(0.98-1.00)$ & $1.00(0.98-1.00)$ \\
\hline
\end{tabular}

Abbreviations: AUC, area under receiver operating characteristic curve; $\mathrm{RBC}$, red blood cell; $\mathrm{Cl}$, confidence interval. 
Table 3. RBC morphological category agreement rates and one grade difference agreement between CellaVision DM9600 and manual microscopic examination

\begin{tabular}{|c|c|c|c|c|}
\hline \multirow{3}{*}{ Poikilocytes } & \multicolumn{2}{|c|}{ Grade agreement } & \multicolumn{2}{|c|}{ One grade difference agreement } \\
\hline & Pre-classification & Post-classification & Pre-classification & Post-classification \\
\hline & Agreement rate (\%) (95\% Cl) & Agreement rate (\%) (95\% Cl) & Agreement rate (\%) $(95 \% \mathrm{Cl})$ & Agreement rate (\%) (95\% Cl) \\
\hline Acanthocytes & $82.5(76.7-87.1)$ & $81.2(75.3-86.0)$ & $99.1(96.5-99.8)$ & $99.1(96.5-99.8)$ \\
\hline Echinocytes & $35.9(29.7-42.6)$ & $46.2(39.6-53.0)$ & $82.1(76.3-86.7)$ & $86.1(80.7-90.2)$ \\
\hline Elliptocytes & $63.7(57.0-69.9)$ & $62.8(56.0-69.1)$ & $100(97.9-100)$ & $99.6(97.1-100)$ \\
\hline Schistocytes & $44.8(38.2-51.6)$ & $54.7(47.9-61.3)$ & $66.8(60.2-72.9)$ & $77.6(71.4-82.8)$ \\
\hline Spherocytes & $89.7(84.7-93.2)$ & $90.1(85.3-93.6)$ & $96.0(92.1-98.0)$ & $97.3(94.0-98.9)$ \\
\hline Target cells & $79.8(73.8-84.8)$ & $85.2(79.7-89.5)$ & $98.2(95.2-99.4)$ & $99.6(97.1-100)$ \\
\hline Tear drop cells & $57.4(50.6-63.9)$ & $67.3(60.6-73.3)$ & $100(97.9-100)$ & $100(97.9-100)$ \\
\hline
\end{tabular}

Abbreviations: $\mathrm{RBC}$, red blood cell; $\mathrm{Cl}$, confidence interval.

highlighted. Regarding the grading of RBC morphological abnormalities, standardization of RBC grading according to consensus guidelines has been recommended [12]. We evaluated the diagnostic performance of the CellaVision Advanced RBC Application with respect to RBC morphological abnormality detection and grading in accordance with the 2015 ICSH guidelines [7]. We compared the manual microscopy results with the results obtained using the CellaVision Advanced RBC Application in both pre- and post-classification modes.

Schistocyte identification is important in blood cell morphology examination, especially in the diagnosis of MAHA [11]. Therefore, the ICSH guidelines recommend schistocyte identification and reporting even at $<1 \%$ for the diagnosis and follow-up of thrombotic thrombocytopenic purpura and hemolytic uremic syndrome [7]. Strict application of the ICSH recommendations and considering schistocytes as positive (reportable) when they represent the main morphological RBC abnormality would increase the specificity to $99.0 \%$ at the expense of a low sensitivity of $42.9 \%$ [13]. We believe that each laboratory should set the optimal cut-off percentage for schistocytes to maximize the detection of MAHA patients, as well as to minimize the number of samples for reclassification. Of the 10 samples with reportable acanthocytes (all grade 2), the acanthocyte grade was underestimated in nine samples (grade 1), as some of these were classified as schistocytes by CellaVision DM9600. Moreover, some acanthocytes that overlapped with other RBC morphologies in the images were not classified into any of the categories (Fig. 2). We believe that a precise differentiation between acanthocytes and schistocytes would increase the sensitivity and specificity for acanthocyte and schistocyte detection, respectively.
The image quality obtained with the CellaVision DM9600 system was crucial for the detection of the RBC morphological categories, consistent with the results of previous studies [14], especially with respect to echinocytes and spherocytes. In the case of echinocytes, the CellaVision DM9600 system tended to over-estimate the grade or provide false detection compared with manual microscopy, although the results were verified by the user. This discrepancy could reflect the limited field examination using the CellaVision DM9600. Echinocytes are often an artefact and may be differently observed from field-to-field. During the manual microscopic examination, the observer selects the optimal fields for RBC morphology examination. In contrast, the CellaVision DM9600 system provides one image obtained from the optimal field that is selected by the software. Providing more images from other optimal areas may enhance echinocyte detection specificity and agreement. With respect to spherocytes, the quality of the PB smear stain and the field in which the image is acquired by the CellaVision DM9600 system are important for detection, because of their characteristic morphological features such as small and dense spherical RBCs that lack central pallor [15].

Some studies have evaluated the sensitivity and specificity of the Advanced RBC Application using various cut-off percentages including those based on the distribution of the control population, those determined using the ROC curve, and the clinical cut-off percentages $[5,11]$. We used the cut-off percentages recommended by the 2015 ICSH guidelines to define 'positive' RBC morphological abnormalities, which hindered a direct comparison of the detected sensitivity and specificity [7]. However, our results are consistent with those of previous studies [5, 11]. Hervent, et al. [11] reported very high sensitivity, but poor speci- 


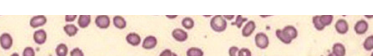

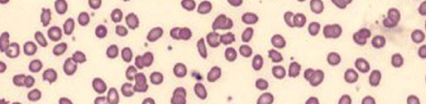

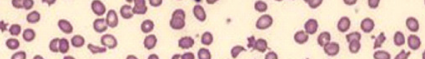

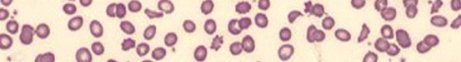

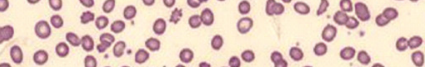

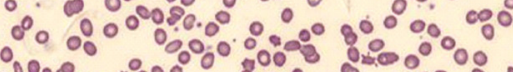

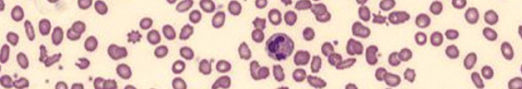

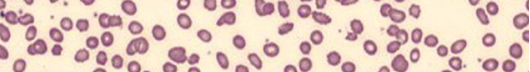

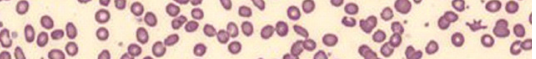

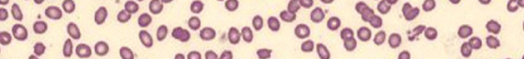
$8: 0600000.0 \%$

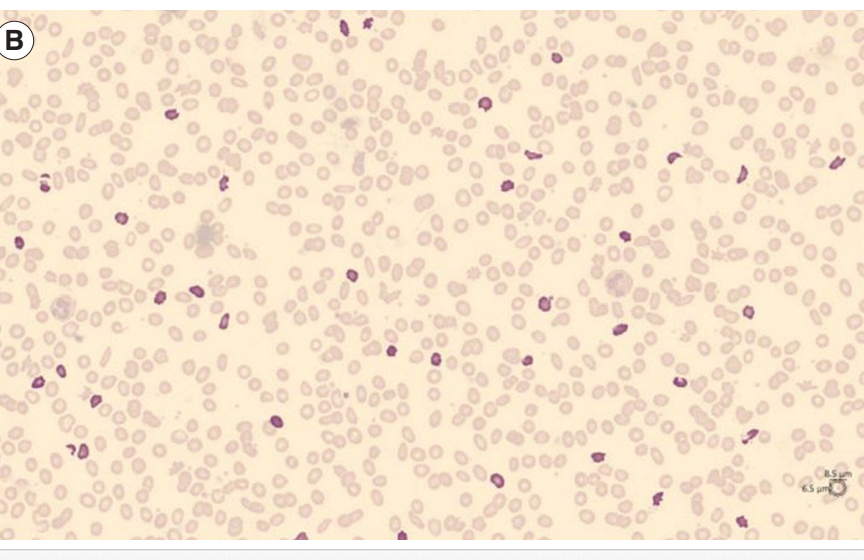
Oindiduatcellu Tarevet cells (10) 0000000000 Schistocytes (98)

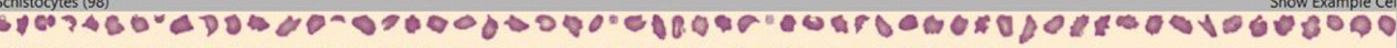

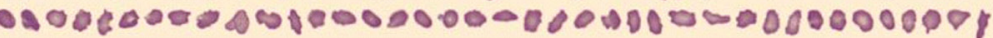

Sickle cells (10)
1030 00000

Elliptogtes (181) Show Example Ce

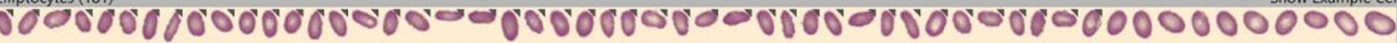
000000000000000000000100000000000000000000000000000001 000000000000000000000000000000000000000000000000000001 00000000000000000

0000000000000000000000000000000000

Acanthocytes (30)

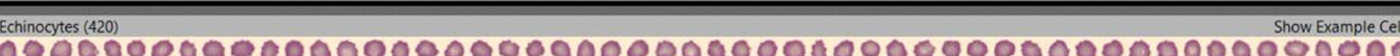
00000000000000000000000000000000000000000000000100004 $0000000000001000000000000000000000 \% 00000000000000000$ 0,000000000000000000000000000400000000000000000000000 00000000000000000000000000000000000000000000000000004 $00000000000000000000800000000000000 m 0100000001000000$

Fig. 2. Screenshots of the CellaVision Advanced RBC Application. (A) The overview image of a sample with acanthocytes (grade 2). (B) Pre-classification results showing some acanthocytes classified as schistocytes. (C) Image of individual cells showing that the acanthocyte grade was underestimated.

Abbreviation: RBC, red blood cell.

ficity, with respect to schistocyte detection using pre-classification software, which was improved after re-classification by the operator. The cut-off percentages determined using the ROC curve for pre- and post-classification were $2.4 \%$ and $0.6 \%$, respectively, and applying these values, they obtained a pre-classification sensitivity and specificity of $77.8 \%$ and $93.1 \%$ and a post-classification sensitivity and specificity of $100 \%$ and $94.8 \%$, respectively. Moreover, difficulties in spherocyte detection have been previously described [5]. Applying a cut-off percentage of $0.1 \%$ (determined by the ROC curve) increased the sensitivity from $0 \%$ to $66.7 \%$; however, spherocyte detection sensitivity was one of the lowest among the RBC morphological abnormalities [5].
Our study showed higher sensitivities in target cells, elliptocytes and tear-drop cells compared to previous study, which evaluated Sysmex DI-60 according to the ICSH grading criteria [8]. Sysmex DI-60 utilizes the same software and modified hardware as CellaVision DM1200 [12]. We evaluated the performance of Advanced RBC Application software using version 7.0.1 updated in 2019. The difference in software version as well as hardware, such as camera lens system or slide making/staining device, may had impact on the different results.

The limitation of this study is that we evaluated the diagnostic performance using a small number of samples that presented (reportable) RBC morphological abnormalities. Relatively few samples had RBC morphological abnormalities exceeding grade 
2. Further extensive evaluations using a large number of positive samples are required to apply the semi-quantitative cut-off values in accordance with the ICSH guidelines [7].

In conclusion, our data indicate that the Advanced RBC Application of CellaVision DM9600 system could provide a valuable screening tool for detecting tear drop cells and target cells, when using the cut-off percentages provided by the $2015 \mathrm{ICSH}$ guidelines [7]. Moreover, despite the rather low specificity, this software could be used for screening and flagging schistocytes when proper cut-off percentages are selected, owing to its high sensitivity.

\section{ACKNOWLEDGEMENTS}

None.

\section{AUTHOR CONTRIBUTIONS}

JAK and SYY contributed to the conception and design of the study; SJP was involved in the clinical evaluation; SJP, JAK, and SYY interpreted the results; JY, JAK, and SYY analyzed the data using statistical analysis tools; JY and JAK drafted the manuscript; and SYY supervised the study. All authors read and approved the final manuscript.

\section{CONFLICTS OF INTEREST}

No potential conflicts of interest relevant to this paper were reported.

\section{RESEARCH FUNDING}

This study was supported by a grant from the Korean Health Technology R\&D Project, Ministry of Health \& Welfare, Korea (H15C1512) and partly supported by CellaVision AB. The funders had no role in study design, data collection, and interpretation or in the decision to submit the work for publication.

\section{ORCID}

$\begin{array}{ll}\text { Seong Jun Park } & \text { https://orcid.org/0000-0002-7158-2509 } \\ \text { Jung Yoon } & \text { https://orcid.org/0000-0001-9296-5085 }\end{array}$

Jung Ah Kwon

https://orcid.org/0000-0001-5321-7279

Soo-Young Yoon

\section{REFERENCES}

1. Stouten K, Riedl JA, Levin MD, van Gelder W. Examination of peripheral blood smears: performance evaluation of a digital microscope system using a large-scale leukocyte database. Int J Lab Hematol 2015;37:e13740.

2. Lee LH, Mansoor A, Wood B, Nelson H, Higa D, Naugler C. Performance of CellaVision DM96 in leukocyte classification. J Pathol Inform 2013;4:14.

3. Cornet E, Perol JP, Troussard X. Performance evaluation and relevance of the CellaVision DM96 system in routine analysis and in patients with malignant hematological diseases. Int J Lab Hematol 2008;30:536-42.

4. Kratz A, Bengtsson HI, Casey JE, Keefe JM, Beatrice GH, Grzybek DY, et al. Performance evaluation of the CellaVision DM96 system: WBC differentials by automated digital image analysis supported by an artificial neural network. Am J Clin Pathol 2005;124:770-81.

5. Criel M, Godefroid M, Deckers B, Devos H, Cauwelier B, Emmerechts J. Evaluation of the red blood cell advanced software application on the CellaVision DM96. Int J Lab Hematol 2016;38:366-74.

6. Horn CL, Mansoor A, Wood B, Nelson H, Higa D, Lee LH, et al. Performance of the CellaVision DM96 system for detecting red blood cell morphologic abnormalities. J Pathol Inform 2015;6:11.

7. Palmer L, Briggs C, McFadden S, Zini G, Burthem J, Rozenberg G, et al. ICSH recommendations for the standardization of nomenclature and grading of peripheral blood cell morphological features. Int J Lab Hematol 2015;37:287-303.

8. Kim HN, Hur M, Kim H, Kim SW, Moon HW, Yun YM. Performance of automated digital cell imaging analyzer Sysmex DI-60. Clin Chem Lab Med. 2017;56:94-102.

9. Rümke CL, Bezemer PD, Kuik DJ. Normal values and least significant differences for differential leukocyte counts. J Chron Dis 1975;28:661-8.

10. Barnes PW, McFadden SL, Machin SJ, Simson E, international consensus group for hematology. The international consensus group for hematology review: suggested criteria for action following automated $\mathrm{CBC}$ and WBC differential analysis. Lab Hematol 2005;11:83-90.

11. Hervent AS, Godefroid M, Cauwelier B, Billiet J, Emmerechts J. Evaluation of schistocyte analysis by a novel automated digital cell morphology application. Int J Lab Hematol 2015;37:588-96.

12. Kratz A, Lee SH, Zini G, Riedl JA, Hur M, Machin S. Digital morphology analyzers in hematology: ICSH review and recommendations. Int J Lab Hematol. 2019;41:437-47.

13. Zini G, d'Onofrio G, Briggs C, Erber W, Jou JM, Lee SH, et al. ICSH recommendations for identification, diagnostic value, and quantitation of schistocytes. Int J Lab Hematol 2012;34:107-16.

14. Yoon J, Kwon JA, Yoon SY, Jang WS, Yang DJ, Nam J, et al. Diagnostic performance of CellaVision DM96 for Plasmodium vivax and Plasmodium falciparum screening in peripheral blood smears. Acta Trop 2019; 193:7-11.

15. Briggs C, Longair I, Slavik M, Thwaite K, Mills R, Thavaraja V, et al. Can automated blood film analysis replace the manual differential? An evaluation of the CellaVision DM96 automated image analysis system. Int J Lab Hematol 2009;31:48-60. 